\title{
One Minute Wonder - Fortbildung während der Arbeitszeit
}

\section{Evaluationsstudie nach Implementierung von One Minute Wonder auf Intensivstationen}

\author{
Lars Krüger $^{1} \cdot$ Thomas Mannebach $^{1} \cdot$ Franziska Wefer $^{1} \cdot$ Christina Bolte $^{1}$ \\ Eingegangen: 2. Januar 2020 / Angenommen: 8. Februar 2021 / Online publiziert: 9. März 2021 \\ (c) Der/die Autor(en) 2021
}

\section{Zusammenfassung}

Hintergrund Fortbildung von Pflegenden hat auf Intensivstationen (ITS) eine hohe Bedeutung. One Minute Wonder (OMW) als Fortbildungsmethode sind dabei so aufbereitet, dass sie in einer Minute gelesen werden können. Bisher wurde die Umsetzung von OMW nur in 2 Untersuchungen in Deutschland mit kleinen Kohorten evaluiert.

Ziel Das primäre Ziel der Evaluationsstudie war zu untersuchen, ob Pflegende auf den ITS OMW zur Fortbildung nutzen. Gleichermaßen sollte aufgezeigt werden, ob OMW zu einem subjektiv beurteilten Bildungsgewinn bei Pflegenden führen. Als sekundäre Ziele sollten Optimierungspotenziale, die Bereitschaft, selbst ein OMW zu erstellen, und die Gesamtbewertung ermittelt werden.

Methode Die Evaluationsstudie wurde als quantitative Vollerhebung $(n=379)$ mittels standardisierten Fragebogens auf allen ITS des Herz- und Diabeteszentrums NRW (HDZ NRW) von September bis Oktober 2018 durchgeführt. Zur Datenauswertung sind Methoden der deskriptiven Statistik angewendet worden.

Ergebnisse Bei einem Rücklauf von 49,87\% $(n=189)$ bestätigten die Pflegenden mit 91,53\% $(n=173)$, dass sie Wartezeiten im Pflegealltag zum Lesen von OMW nutzen. 73,55\% $(n=139)$ gaben an, dass die Informationen aus den OMW im Praxisalltag hilfreich sind. Als Wechselintervall wurde mit 69,31\% ( $n=131)$ 14-tägig ausgewählt. Die Bereitschaft, selbst ein OMW zu erstellen, war ausgewogen. Die Methode wurde im Median mit $2(40,21 \%, n=76)$ und im Mittelwert mit $1,90(n=184)$ bewertet.

Schlussfolgerung Die OMW stellen für Pflegende eine sinnvolle ergänzende Fortbildungsmethode dar. Um den Wissenstransfer durch OMW empirisch zu belegen, ist weitere Forschung nötig.

Schlüsselwörter Berufliche Praxis · Innerbetriebliche Fortbildung · Intensivstation · Pflege · Wissenstransfer

\section{One minute wonder-Inservice training during working hours}

Evaluation study after implementation of one minute wonder in intensive care units

\begin{abstract}
Background Further training of nurses is very important for working in an intensive care unit. One minute wonder (OMW) as a training method are prepared in such a way that they can be read within $1 \mathrm{~min}$. To date, the implementation of OMW has only been evaluated two times in Germany with small cohorts.

Aim The primary aim of the evaluation study was to determine if nurses in intensive care wards use OMW for further training and to demonstrate whether nurses have a subjective knowledge benefit while using OMW. Secondary aims for this trial were to research the potential for optimization, willingness for preparing an OMW and overall evaluation.
\end{abstract}

Lars Krüger

lkrueger@hdz-nrw.de

1 Herz- und Diabeteszentrum NRW,

Universitätsklinik der Ruhr-Universität

Bochum, Georgstraße 11, 32545 Bad Oeynhausen,

Deutschland 
Method This evaluation study was conducted as a quantitative census $(n=379)$ from September to October 2018 with standardized questionnaires in all intensive care units of the Heart and Diabetes Center North-Rhine/Westphalia (Herzund Diabeteszentrum NRW, HDZ NRW). For data analysis, descriptive methods were used.

Results With a return of $49.87 \%(n=189), 91.53 \%(n=173)$ of the participants confirmed that they use waiting periods to read OMW. Of the participants $73.55 \%(n=139)$ mentioned that the information of the OMW was useful in everyday practice. The participants selected a 14-day change interval with $69.31 \%(n=131)$. The willingness for preparing an OMW was balanced. The median of the method was rated with $2(40.21 \%, n=76)$ and the arithmetic average was $1.90(n=184)$. Conclusion The OMW is a useful supplementary training method for nursing staff. To confirm the transfer of knowledge by OMW further research is necessary.

Keywords Intensive care units $\cdot$ Further education $\cdot$ Knowledge nursing $\cdot$ Professional practice

\section{Hintergrund}

Aufgrund steigender Arbeitsintensität und -komplexität in der Pflege ist eine effektive Wissensvermittlung sowohl für Arbeitnehmende als auch für Arbeitgebende von Interesse (Fessele et al. 2018; Krüger und Mannebach 2018). Um auf die veränderten Anforderungen zu reagieren, sind speziell ausgewählte Aus- und Weiterbildungsangebote notwendig (Fessele et al. 2018). Ziel ist es, eine qualitativ hochwertige pflegerische Versorgung durch eine Kompetenzentwicklung zu gewährleisten. Durch den Fortschritt, z.B. in der Pflegewissenschaft, werden heutzutage neue Erkenntnisse schneller generiert und veröffentlicht. Das sich stetig entwickelnde Wissen muss jedoch so aufbereitet werden, dass Beschäftige dies in der Praxis zeitnah aufnehmen, nutzen und anwenden können. So wird in einer Studie zur Anwendung von externer Evidenz in der Pflege u.a. darauf verwiesen, dass eine „einfache Verfügbarkeit von aufbereiteten Forschungsergebnissen“ (Haselinger-Baumann et al. 2015) eine Auswirkung auf deren Anwendung in der Praxis hat.

Die Fort- und Weiterbildung sollte sich diesen Herausforderungen stellen und nutzerorientierte Angebote entwickeln und ausbauen. In Krankenhäusern stehen heutzutage verschiedene Fortbildungsmethoden zur Verfügung, wie z.B. klassische Präsenzveranstaltungen, „Whiteboard teaching“ (Fessele et al. 2018) oder E-Learning-Angebote (Knoppik 2004). Diese Angebote können unterschiedliche Schwerpunkte, wie z.B. Persönlichkeitsentwicklung oder fachliche Kompetenz, aufweisen.

Die bisher etablierten Methoden haben oftmals gemeinsam, dass die Beschäftigten zusätzlich zur regulären Arbeitszeit zeitliche Ressourcen zur Verfügung stellen müssen, um an diesen Veranstaltungen teilnehmen zu können (Krüger und Mannebach 2018). Dies kann beispielsweise durch ein Selbststudium vor und nach Angeboten mit Präsenzpflicht erfolgen (Knoppik 2004). Eine weitere Möglichkeit besteht in der Durchführung von abteilungsinternen Fortbildungen innerhalb der regulären Arbeitszeit. Diese sind in der Regel kurz, jedoch erfordern sie oft Organisationsbemühungen seitens der Pflegenden, für eine Vertretung in der Patientinnen- und Patientenversorgung zu sorgen (Brunnhuber 2008, S. 46). Aufgrund begrenzter zeitlicher und personeller Ressourcen sind abteilungsinterne Fortbildungen innerhalb der regulären Arbeitszeit mitunter nicht regelhaft zu gewährleisten.

Um das Fortbildungsprogramm zu ergänzen, wurde 2015 im Herz- und Diabeteszentrum NRW (HDZ NRW) nach alternativen Fortbildungsmethoden recherchiert. Hierbei wurden die sogenannten One Minute Wonder (OMW) identifiziert (Schmidt und Krüger 2016).

Die aus England stammende Methode nutzt regelmäßig wiederkehrende Wartezeiten in der täglichen Pflegepraxis zur Fortbildung (Rowlinson 2014). In der Intensivpflege können diese in unterschiedlicher Häufigkeit und Dauer, beispielsweise am Blutgasanalyse(BGA)-Gerät, entstehen. An diesen Orten werden pflegerelevante Lernposter präsentiert und in festgelegten Abständen ausgetauscht, sodass abwechselnd verschiedene Themen gelesen werden können. Der entsprechende Inhalt soll von Lesenden innerhalb einer Minute erfasst werden können (Rowlinson 2014; Schmidt und Krüger 2016). Dies erfolgt während der regulären Arbeitszeit und somit ohne zusätzliches zeitliches Aufkommen. Ähnlich dem Whiteboard teaching (Fessele et al. 2018) werden die Inhalte kurz und prägnant auf einem Medium aufbereitet (Rowlinson 2014). Der Fokus dieser Methode liegt in der Erweiterung von fachlicher Kompetenz und somit auf einer Wissensvermittlung.

Eine Evaluation seitens der Entwicklerin wurde in der Vergangenheit nicht durchgeführt.

Im Rahmen einer weiteren Literaturrecherche ließ sich international keine ausreichende weiterführende Datenlage ausfindig machen. Lediglich 2 Pilotprojekte aus den Niederlanden (Jonker et al. 2016) und England (Bray 2016), in denen OMW als Fortbildungsmethode eingesetzt wurden, konnten identifiziert werden. Die Verwendung von OMW wurden von den Pflegenden als hilfreich wahrgenommen (Jonker et al. 2016) und führte in Kombination mit weiteren 
Fortbildungsmethoden zu einer Verbesserung der Patientinnen- und Patientenversorgung (Bray 2016). Bisher liegen deutschlandweit ausschließlich 2 publizierte Untersuchungen mit jeweils kleinen Kohorten vor. Hierbei handelt es sich um Pilotevaluationen auf Intensivstationen aus dem HDZ NRW ( $n=45)$ und dem Universitätsklinikum Schleswig-Holstein (UKSH, $n=55$ ) nach Einführung von OMW (Krüger 2017; Lehnen et al. 2019). Dabei stellte sich im Kern heraus, dass sich in der pflegerischen Praxis im HDZ NRW 44,40\% der Pflegenden an die Inhalte eines OMW erinnerten (Krüger 2017). Auf insgesamt 2 Pilotstationen im UKSH waren die Ergebnisse heterogen. So gaben in einer Abteilung 80,00\% der befragten Pflegenden an, sich im Praxisalltag an Inhalte eines OMW erinnert zu haben, während in der anderen Abteilung 32,50\% diese Angabe machten (Lehnen et al. 2019). Die Autorschaften beider Publikationen werteten die Ergebnisse positiv.

Nach der erfolgreichen Pilotevaluation wurde die Methode im HDZ NRW auf allen Intensivstationen umgesetzt.

Um die Datenlage zu erweitern und eine größere Kohorte zu befragen, wurde eine Vollerhebung auf allen Intensivstationen des HDZ NRW durchgeführt.

\section{Ziel}

Das primäre Ziel der Evaluationsstudie war zu untersuchen, ob Pflegende auf den Intensivstationen OMW zur Fortbildung nutzen. Gleichermaßen sollte mit einer selbst konstruierten Skala aufgezeigt werden, ob OMW zu einem subjektiv beurteilten Bildungsgewinn bei Pflegenden führen. Das sekundäre Ziel bestand in der Identifikation möglicher Optimierungspotenziale, sodass stationsbezogene Anpassungen durchgeführt werden können. Zusätzlich sollten die Bereitschaft, selbst ein OMW zu erstellen, sowie eine Gesamtbewertung ermittelt werden.

\section{Methode}

\section{Design}

Zur Beantwortung der Fragestellung wurde eine quantitative Evaluationsstudie mithilfe eines selbst konstruierten Fragebogens im HDZ NRW durchgeführt.

\section{Setting und Stichprobe}

Das HDZ NRW verfügt über 500 Planbetten, davon sind 105 Intensivbetten. Die Klinik betreibt insgesamt 6 Intensivstationen mit den Fachgebieten Thorax- und Kardiovaskularchirurgie, inkl. Herztransplantation, Kardiologie sowie Kinderkardiologie und -herzchirurgie. Zum Zeitpunkt der
Datenerhebung waren auf den Intensivstationen im HDZ NRW 384 Pflegende beschäftigt. Seit 2016 werden im HDZ NRW als Methode zur Wissensvermittlung eingesetzt. Zunächst erfolgte ein Pilotprojekt auf einer Intensivstation. Eine Evaluation der Projektumsetzung fand ebenfalls 2016 statt. Im Jahr 2017 wurde das Konzept auf alle Intensivstationen ausgeweitet. Zur Festigung der Umsetzung wurden auf jeder Station OMW-beauftragte Pflegefachpersonen benannt. Ein Wechsel der OMW fand seit 2017 14-tägig statt. Dies war ein Ergebnis der Pilotevaluation von 2016 (Krüger 2017).

$\mathrm{Zu}$ dieser Befragung sind alle Pflegenden der Intensivstationen mit mindestens einer grundständigen 3-jährigen Ausbildung $(n=379)$ des HDZ NRW, inklusive des Intensivpflegepools, eingeladen worden. Ausgeschlossen wurden die OMW-beauftragten Pflegefachpersonen $(n=5)$ der Intensivstationen, da diese am Pretest des Fragebogens teilgenommen haben.

\section{Datenerhebung}

Die Datenerhebung erfolgte über insgesamt 8 Wochen von Anfang September bis Ende Oktober 2018. Zu diesem Zeitpunkt waren OMW bereits über $1 \mathrm{Jahr}$ in den eingeschlossenen Abteilungen etabliert. Die Fragebögen und ein Anschreiben zur Erläuterung der Studie wurden in gedruckter Form und einem geschlossenen Umschlag durch die Studienleitungen über die Postfächer der Abteilungen an die Pflegenden verteilt. Zur Information der Beteiligten wurden zusätzlich Poster zur Befragung in den betroffenen Abteilungen gut sichtbar ausgehängt und E-Mails an alle Pflegenden versendet. Die ausgefüllten Fragebögen konnten von den Teilnehmenden anonym in eine Box auf den Stationen eingeworfen werden. Ein eingeworfener Fragebogen zählte als freiwillige Zustimmung. Alle Teilnehmenden erhielten 4 Wochen nach dem Start der Erhebung per E-Mail eine Erinnerung an die Befragung.

\section{Erhebungsinstrument}

Der Fragebogen wurde an den zuvor verwendeten Fragebogen zur Pilotevaluation angelehnt (Krüger 2017), da national und international kein Fragebogen zur Forschungsfrage zur Verfügung stand. Es fand eine Erweiterung von ursprünglich 9 Fragen auf 15 Fragen statt, um weitere Kategorien abzubilden. Im Entwicklungsprozess wurde zunächst ein 3-teiliger tabellarischer Aufbau gewählt. Dadurch sollten Kategorie, Indikator und die spezifische Frage getrennt voneinander dargestellt werden (Mayer 2015, S. 192, 193). Im Anschluss entstand der Fragebogen, welcher mehrfach grafisch überarbeitet wurde. Die abschließende Erstellung erfolgte elektronisch mit der Software Teleform. Im Fragebogen kamen sowohl nominalskalierte (Single- und Multi- 
Tab. 1 Fragebogen der Evaluationsstudie „One Minute Wonder - Fortbildung während der Arbeitszeit - Evaluationsstudie nach Implementierung von One Minute Wonder auf Intensivstationen“

\begin{tabular}{ll}
\hline Frage & Antwortmöglichkeit \\
Konkrete Frage im Fragebogen & \\
\hline
\end{tabular}

Weißt du, dass es auf deiner

Abteilung One Minute Wonder $(O M W)$ gibt?

Wie bist du auf die OMW aufmerksam geworden?

An wie vielen Orten würdest du in deiner Abteilung ein OMW aushängen?

Welche Aushangsorte würdest du für OMW wählen? (Mehrfachauswahl möglich)

In welchen zeitlichen Abständen würdest du ein OMW wechseln?

Nutzt du Wartezeiten im Dienst zum Lesen von OMW?

Welches OMW (Thema) fandest du besonders gut gelungen?

„Bei einer konkreten pflegerischen Handlung hat mir die Information aus einem OMW schon einmal geholfen." Dieser Aussage stimme ich ...

Wie häufig kommt es vor, dass Inhalte aus einem OMW direkt in der Praxis nützlich sind?

Würdest du die bereits ausgehängten OMW gerne später noch einmal nachlesen?

Falls ja: In welcher Form möchtest du die OMW nachlesen?

Hast du schon einmal ein OMW erstellt?

Welche OMW-Themen würdest du dir wünschen? (Bitte kurz nennen)

Würdest du in Zukunft selbst OMW erstellen? (Bitte ankreuzen)

„OMW als Fortbildungsmethode in der Pflege bewerte ich insgesamt als ..."
Ja; Nein

Aushänge von OMW in der Abteilung; Informationen durch MitarbeiterInnen

Im Rahmen der Einarbeitung Sonstiges (bitte kurz nennen)

1; 2; 3; mehrere (bitte Anzahl nennen)

BGA-Gerät; PatientInnenküche; Personalküche; Stationszentrale; Sonstige (bitte nennen)

Alle 7 Tage; alle 14 Tage; sonstige Zeitangabe in Tagen (bitte nennen)

Likert-Skala 1-6 (sehr oft bis gar nicht)

Freitextantwort

Likert-Skala 1-6 (voll zu bis gar nicht zu)

Likert-Skala 1-6 (sehr oft bis gar nicht)

Ja; Nein

Ausgedruckt (z. B. in einem Ordner); als .pdf-Datei in einer Datenbank (z. B. Moodle); Sonstiges

Ja; Nein

Freitextantwort

Likert-Skala 1-6 (Ja, auf jeden Fall bis Nein, auf keinen Fall)

Likert-Skala 1-6 (sehr gut bis ungenügend)
Tab. 1 (Fortsetzung)

\begin{tabular}{ll}
\hline $\begin{array}{l}\text { Frage } \\
\text { Konkrete Frage im Fragebogen }\end{array}$ & Antwortmöglichkeit \\
\hline $\begin{array}{l}\text { Zusätzlich möchte ich noch Fol- } \\
\text { gendes zum Thema OMW sagen: }\end{array}$ & Freitextantwort \\
$\begin{array}{l}\text { Abschließend bitten wir, doch } \\
\text { noch Fragen zu deiner Person zu }\end{array}$ & \\
beantworten: & \\
$\begin{array}{l}\text { In welcher Abteilung arbeitest du } \\
\text { aktuell? }\end{array}$ & Auflistung der Abteilungen \\
$\begin{array}{l}\text { Über wie viele Jahre Berufser- } \\
\text { fahrung verfügst du? }\end{array}$ & Unter 2 Jahre; 2 bis 5 Jahre; \\
\hline
\end{tabular}

BGA Blutgasanalyse

ple-Choice-Fragen) als auch ordinalskalierte Variablen (6stufige Likert-Skala) zum Einsatz. Zusätzlich waren freie Antworten auf offene Fragestellungen möglich. Vor Beginn der Erhebung wurde ein standardisierter Pretest des erweiterten Fragebogens mit insgesamt 8 Personen durchgeführt, 5 Personen davon waren OMW-beauftragte Pflegende der Intensivstationen. Alle am Pretest teilnehmenden Personen verfügten über unterschiedliche Berufserfahrungen in der kardiochirurgischen, kardiologischen und pädiatrischen Intensivpflege sowie der Transplantationspflege und erstellten z.T. bereits OMW. Der Fragebogen wurde auf Verständlichkeit, zeitlichen Aufwand und mögliche fehlende Aspekte geprüft. Nach dem Pretest sind Korrekturen bezüglich der Grammatik am Fragebogen vorgenommen worden. Eine Darstellung des Fragebogens ist in Tab. 1 einzusehen.

\section{Datenanalyse}

Für die statistische Analyse ist das Softwareprogramm Microsoft ${ }^{\circledR}$ Excel verwendet worden. Die Dateneingabe erfolgte elektronisch mit der Software Teleform. Die Daten wurden bei unklarer Markierung im Fragebogen anschließend manuell kontrolliert. Freitexte wurden händisch übertragen. Alle Fragebögen, bei denen mindestens $50 \%$ der Fragen ausgefüllt wurden, sind in der Analyse berücksichtigt worden. Fehlende Daten wurden in der Ergebnisdarstellung durch separat dargestellte $n$ ausgewiesen.

Es wurde ein deskriptives Auswertungsverfahren angewendet. Die Variablen sind in Abhängigkeit des Datenniveaus abgebildet worden. Es kamen Häufigkeitsverteilungen (absolut, relativ) sowie Lagekennzahlen (Median und arithmetisches Mittel) zur Anwendung.

\section{Ethische Überlegungen und Berichterstattung}

Die Teilnahme an der Studie war freiwillig. Die Studie wurde durch die Ethikkommission der Medizinischen Fakultät der Ruhr-Universität Bochum (Aktenzeichen 2018-398) begutachtet. Die Berichterstattung erfolgt in Anlehnung an das STROBE-Statement (Von Elm et al. 2008). 
Tab. 2 Nutzung von Wartezeiten durch Pflegende zum Lesen eines One Minute Wonder auf der Intensivstation

\begin{tabular}{|c|c|c|c|c|c|c|c|}
\hline \multicolumn{8}{|c|}{$\begin{array}{l}\text { „Nutzt Du Wartezeiten im Dienst zum Lesen von OMW?“ } \\
\text { Likert-Skala } 1-6(1=\text { sehr oft, } 6=\text { gar nicht })\end{array}$} \\
\hline & 1 & 2 & 3 & 4 & 5 & 6 & $\mathrm{k} / \mathrm{a}$ \\
\hline$n=$ & 38 & 75 & 60 & 7 & 7 & 0 & 2 \\
\hline$\%=$ & 20,11 & 39,68 & 31,75 & 3,70 & 3,70 & 0,00 & 1,06 \\
\hline
\end{tabular}

Tab. 3 Häufigkeit, in der Pflegende die Inhalte eines One Minute Wonder in der Praxis als nützlich ansehen

„Wie häufig kommt es vor, dass Inhalte aus dem OMW direkt in der Praxis nützlich sind?“

Likert-Skala $1-6(1=$ sehr oft, $6=$ gar nicht $)$

\begin{tabular}{llllllll}
\hline & 1 & 2 & 3 & 4 & 5 & 6 & $\mathrm{k} / \mathrm{a}$ \\
\hline$n=$ & 24 & 81 & 60 & 12 & 3 & 1 & 8 \\
$\%=$ & 12,70 & 42,86 & 31,75 & 6,35 & 1,59 & 0,53 & 4,23 \\
\hline
\end{tabular}

Tab. 4 Häufigkeit, in denen Pflegenden die Inhalte eines One Minute Wonder in der Praxis geholfen haben

„Bei einer konkreten pflegerischen Handlung hat mir die Information aus einem OMW schon einmal geholfen. Dieser Aussage stimme ich:“

Likert-Skala $1-6(1=$ stimme voll zu, $6=$ stimme gar nicht $z u)$

\begin{tabular}{lllllllll}
\hline & 1 & 2 & 3 & 4 & 5 & 6 & $\mathrm{k} / \mathrm{a}$ \\
\hline$n=$ & 19 & 49 & 71 & 17 & 19 & 3 & 11 \\
$\%=$ & 10,05 & 25,93 & 37,57 & 8,99 & 10,05 & 1,59 & 5,82 & \\
\hline
\end{tabular}

\section{Ergebnisse}

Es wurden insgesamt 379 Fragebogen verteilt. Die Rücklaufrate betrug 49,87\% ( $n=189)$. 14,81\% der Befragten gaben an, weniger als 2 Jahre Berufserfahrung zu haben. Knapp ein Viertel der Pflegenden $(34,24 \%)$ war 2 bis 5 Jahre im Beruf und über die Hälfte $(59,26 \%)$ länger als 5 Jahre. 1,59\% machten hier keine Angaben. Fast alle Befragten $(96,83 \%, n=183)$ bestätigten, dass ihnen die Methode der OMW bekannt ist. Bei der Frage, ob sie Wartezeiten zum Lesen eines OMW nutzen, gaben 91,53\% $(n=173)$ der Befragten auf einer Likert-Skala von 1 (sehr oft) bis 6 (gar nicht) die Zahlen 1-3 an (Tab. 2).

\section{Subjektiv beurteilter Bildungsgewinn}

Die Frage, wie oft Informationen auf einem OMW in der Praxis nützlich sind, beantworteten $87,30 \%(n=165)$ der Pflegenden auf einer Likert-Skala von 1 (sehr oft) bis 6 (gar nicht) mit den Zahlen 1-3 (Tab. 3).

Rund drei Viertel $(73,55 \%, n=139)$ der Teilnehmenden stimmten der Aussage „Bei einer konkreten pflegerischen Handlung hat mir die Information aus einem OMW schon einmal geholfen“ auf einer Likert-Skala von 1 (stimme voll $\mathrm{zu}$ ) bis 6 (stimme gar nicht $\mathrm{zu}$ ) mit den Zahlen 1 bis $3 \mathrm{zu}$ (Tab. 4).

\section{Optimierungspotenzial}

Im Rahmen der Evaluationsstudie wurden verschiedene Punkte erhoben, die den Einsatz eines OMW in der Praxis aus Sicht der Teilnehmenden möglicherweise verbessern können. Zur gewünschten Zahl der Präsentationsorte gab knapp die Hälfte $(47,09 \%, n=89)$ der Pflegenden 3 Orte an, gefolgt von 2 Aushangorten mit 29,10\% $(n=55)$.

Bei der Frage nach geeignet erscheinenden Plätzen für OMW wurden mit dem BGA-Gerät und der Patientinnenund Patientenküche primär die bereits verwendeten Orte genannt, aber auch einige Alternativen. Dazu gehörten z. B. die Apotheke, das WC oder Schleusen von Zimmern auf der Intensivstation.

Die Frage nach dem präferierten Wechselintervall beantworteten 69,31\% $(n=131)$ der Mitarbeitenden mit einer 14tägigen Zeitspanne, 16,93\% $(n=32)$ würden das Intervall auf 7 Tage reduzieren. Vereinzelt wurden andere Zeiträume zwischen 5 und 30 Tagen gewünscht.

Die Pflegenden wurden befragt, welche Themen bisher für sie am interessantesten waren. Rund 90 verschiedene Antworten entfielen darauf. Das Themenspektrum war dabei heterogen. Genannt wurden u.a. die Pflege von Patientinnen und Patienten mit einer extrakorporalen Membranoxygenierung (ECMO), spezifische Positionierungen und die Verwendung von Verbandmaterial. Circa 80 Vorschläge für zukünftige OMW-Themen wurden abgegeben. Dazu zählten beispielsweise Herzunterstützungssysteme, Intubationsmethoden, Palliativpflege und das akute Lungenversagen.

\section{Interesse an One Minute Wonder}

Nur wenige Befragte $(10,58 \%, n=20)$ gaben an, bereits veröffentlichte OMW später noch einmal nachlesen zu wol- 
len, beispielsweise als Ausdruck in einem Sammelordner oder in digitaler Form.

Die grundsätzliche Bereitschaft, selbst einmal ein oder mehrere OMW zu erstellen wurde, etwa ausgewogen angegeben. $46,04 \%(n=87)$ antworteten auf einer Likert-Skala von 1 bis $6(1=$ auf jeden Fall, $6=$ auf keinen Fall $)$ mit den Zahlen 1 bis 3. Etwa dieselbe Menge $(49,20 \%, n=93)$ verwendete die Zahlen 4-6. Neun Befragte $(4,76 \%)$ machten hierzu keine Angaben.

\section{Gesamtbewertung zu One Minute Wonder}

Die meisten Befragten entschieden sich als abschließende Bewertung der Fortbildungsmethode für die Schulnoten gut $(40,21 \%, n=76)$ und sehr gut $(36,51 \%, n=69)$. Der Median betrug $2(n=76)$, der Mittelwert 1,90 $(n=184)$. Auf die Note befriedigend entfielen $16,40 \%(n=31)$, während ausreichend und mangelhaft mit jeweils $2,12 \%(n=4)$ gewählt wurden. Die Note ungenügend wurde nicht angegeben. Fünf Teilnehmende (2,65\%) beantworteten diese Frage nicht.

\section{Diskussion}

\section{Hauptergebnisse}

In dieser quantitativen Evaluationsstudie sollte untersucht werden, ob Pflegende OMW in der Praxis nutzen. Gleichermaßen war das Ziel zu ermitteln, ob OMW bei den Pflegenden auf Intensivstationen zu einem subjektiv beurteilten Bildungsgewinn führen. Als sekundäre Ziele sollten die Optimierungspotenziale sowie die Bereitschaft, selbst ein OMW zu erstellen, untersucht werden. Insgesamt wurde diese Fortbildungsmethode von den Teilnehmenden positiv bewertet.

Bei einem Rücklauf von rund der Hälfte aller Fragebögen ist die Teilnahme der Pflegenden mit denen der Projektstation $(51 \%, n=45)$ aus dem Jahr 2016 vergleichbar (Krüger 2017). Ähnlich verhält es sich bei der Bekanntheit der Fortbildungsmethode. Die meisten Teilnehmenden gaben ebenfalls an, die Fortbildungsmethode OMW zu kennen (Krüger 2017). Diese Ergebnisse wurden auch in der Untersuchung am UKSH generiert (Lehnen et al. 2019).

Es bestätigte sich zudem, dass Pflegende Wartezeiten im Dienst zum Lesen von OMW nutzen. Dieses Ergebnis wurde in der digitalen Version der OMW von Jonker et al. (2016) ebenfalls dargestellt. Somit bietet es sich in der pflegerischen Praxis für arbeitgebende Betriebe an, ein entsprechendes Angebot von OMW für die interessierten Pflegenden und weitere am Versorgungsprozess beteiligte Mitarbeitende vorzuhalten. Wartezeiten entstehen dabei auch länderübergreifend in Settings der Gesundheitsversor- gung und können von interessierten Mitarbeitenden zu kurzen Fortbildungen genutzt werden.

Ein subjektiv beurteilter Bildungsgewinn durch OMW findet aus Sicht der Befragten statt. Über die Hälfte der Teilnehmenden gaben dazu auf einer 6-stufigen Likert-Skala mit den Stufen 1 und 2 an, dass Inhalte aus einem OMW in der direkten Patientinnen- und Patientenversorgung häufig nützlich sind. Hier stützen die Ergebnisse dieser Studie die von Haselinger-Baumann et al. (2015).

Die Teilnehmenden gaben zusätzlich an, dass ihnen die Informationen aus einem OMW bei einer konkreten pflegerischen Handlung geholfen haben. Auf einer 6-stufigen Likert-Skala $(1=$ stimme voll $\mathrm{zu}$ bis $6=$ stimme gar nicht zu) bestätigten dies rund drei Viertel der Befragten mit den Stufen 1 bis 3. Dieses Ergebnis ist besser als jenes aus der ersten Evaluation von 2016. Hier waren es mit $44 \%$ etwas weniger als die Hälfte der Pflegenden (Krüger 2017). Dabei wurde jedoch eine Erhebung mit dichotomen Antworten $(\mathrm{Ja} / \mathrm{Nein})$ durchgeführt. Somit hatten die Teilnehmenden keine Skala zur Auswahl, sondern mussten sich hier entscheiden, ob die Inhalte eines OMW bei einer pflegerischen Handlung hilfreich waren oder nicht. Pflegerische Situationen, in denen Inhalte möglicherweise nur z. T geholfen haben, könnten dabei in der Einschätzung der Teilnehmenden keine Berücksichtigung gefunden haben.

Die bisher verwendeten und während der Evaluationsstudie präsentierten Lernposter waren thematisch breit aufgestellt. Es wurden beispielsweise Themen zur BGA, die Funktionsweise einer Thoraxdrainage oder auch Studienergebnisse zur Bauchlagerung präsentiert. Darüber hinaus zeigte sich in dieser Studie inhaltlich ein direkter praktischer Bezug zur Pflege in unterschiedlicher Ausprägung. Die Pflegenden wünschen neben den bereits vorhandenen OMW-Themen u. a. auch Informationen zu Medikamenten, Krankheitsbildern, der Medizintechnik und Blutgerinnung. Generell sind zusammengefasste Informationen für Pflegende praxisrelevant. Schniering et al. (2015) stellten ebenso fest, dass sich Pflegende zusammengefasste und komprimierte Informationen wünschen.

Unterschiede zu den Voruntersuchungen gab es in der Anzahl der Aushangorte. Während bei den bisherigen Evaluationen 2 Bereiche präferiert wurden (Krüger 2017; Lehnen et al. 2019), zeigt die aktuelle Erhebung mit rund der Hälfte der Befragten einen Trend zu 3 Aushangorten. Zusätzlich wurden mögliche Wartezonen im Arbeitsalltag von Pflegenden identifiziert, welche zukünftig zielgerichtet pro Station genutzt werden können. Über die Hälfte der Befragten gaben ein 14-tägiges Wechselintervall für OMW an. $\mathrm{Zu}$ demselben Ergebnis kommen auch die vorherigen Evaluationen (Krüger 2017; Lehnen et al. 2019). Das Vorgehen kann daher als erster Hinweis gelten, ein praxisnahes Wechselintervall umzusetzen. Über diesen Zeitraum können Pflegende den Lerninhalt beliebig oft lesen. Dieses Vorgehen 
wird Wiederholungsstrategie genannt und unterstützt das vertiefte Lernen (Löwenstein 2016, S. 29; Frick-Salzmann 2017, S. 31). Lehnen et al. (2019) kommen hier außerdem zu dem Schluss, dass so auch Pflegende mit einem reduzierten Stellenumfang die Gelegenheit erhalten, möglichst viele Lernposter in der Praxis zu nutzen.

OMW stellen aus der Sicht der Befragten anscheinend ein geeignetes ergänzendes Mittel zur Wissensvermittlung in der Praxis dar. Thematisch waren die dargestellten Inhalte bisher breit gefächert und sollten auch zukünftig nicht nur ausschließlich direkten Praxisbezug haben, sondern beispielsweise auch Forschungsergebnisse aus der Pflegewissenschaft beinhalten. Das von Fessele et al. (2018) als praxisnah beschriebene Whiteboard teaching ähnelt dabei dem Vorgehen des OMW. Dazu wird jedoch eine deutlich größere Präsentationsfläche benötigt. Außerdem bleibt offen, inwiefern bei dieser Methode ein Verweis oder Bezug auf Literaturquellen zu den dargestellten Themen sichergestellt wird.

OMW stellen ein Tool im Sinne eines Bottom-up-Systems dar (Krüger und Mannebach 2018). Das Engagement Pflegender, selbst ein OMW zu erstellen, variiert in dieser Evaluationsstudie allerdings und zeigt eine breite Verteilung. Anders zeigen sich die Ergebnisse am UKSH. Rund ein Viertel der Befragten kann sich hier vorstellen, selbst ein OMW zu erstellen (Lehnen et al. 2019). In der ersten Evaluation am HDZ NRW waren es mit etwas mehr als die Hälfte der Teilnehmenden (Krüger 2017). In dieser Untersuchung ist es mit rund der Hälfte der teilnehmenden Pflegenden etwas weniger. Das kann darauf zurückzuführen sein, dass nicht nur innerhalb der Pilotstation evaluiert wurde, sondern eine Vollerhebung auf mehreren Intensivstationen stattfand. Innerhalb des UKSH war die Methode in manchen der an der Evaluation teilnehmenden Abteilungen (Lehnen et al. 2019) noch neu. Möglicherweise hat der Zeitaufwand, welcher bei der ersten Erstellung eines OMW höher ist, als wenn eine gewisse Routine eintritt, zu einer Zurückhaltung seitens der Pflegenden geführt.

Die abschließende Gesamtbewertung fiel positiv aus und spricht ebenfalls für OMW als Methode in der Fortbildung. OMW wurden im Median mit der Zensur ,gut" bewertet und im Mittelwert mit 1,90. Im UKSH bewerten die Pflegenden OMW für sich selbst im Mittelwert mit der Zensur 2,20 und zusätzlich den Nutzen für das Kollegium mit 2,10 (Lehnen et al. 2019).

Die Ergebnisse sind somit nahezu gleich und bekräftigen den positiven Beitrag von OMW im Rahmen von Fortbildungsmethoden.

\section{Limitation}

Im Rahmen der Evaluationsstudie wurde ein selbst konstruierter Fragebogen entwickelt. Es fand keine umfangreiche, jedoch durch den standardisierten Pretest eine inhaltliche Validierung statt. Bei der Frage zur Berufserfahrung in der Pflege wurde dabei nicht darauf verwiesen, dass die Zeit der grundständigen Pflegeausbildung nicht mitgezählt werden soll. Darüber hinaus wurde die Frage „Bei einer konkreten pflegerischen Handlung hat mir die Information aus einem OMW schon einmal geholfen" nicht neutral gestellt. Dies kann zu Verzerrungen in der Beantwortung seitens der Pflegenden geführt haben.

Der Rücklauf von rund der Hälfte aller Fragebögen kann als akzeptabel (Weigl 2016), jedoch nicht umfassend gewertet werden. Die Daten wurden ausschließlich auf den Intensivstationen des HDZ NRW mit den Fachrichtungen Thorax- und Kardiovaskularchirurgie, Herztransplantation, Kardiologie sowie Kinderkardiologie und -herzchirurgie erhoben. Somit sind die Ergebnisse nicht ohne Weiteres auf andere Kliniken/Fachbereiche zu übertragen. In den bisherigen Evaluationen (Krüger 2017; Lehnen et al. 2019) mit kleinen Kohorten wurde ein ähnliches Ziel verfolgt, wie in dieser Evaluationsstudie. Dennoch galt es hier, eine größere Kohorte über mehrere Abteilungen hinweg zu generieren, um aussagekräftigere Daten zu erhalten.

\section{Stärken}

In dieser Studie wurde die bisher größte Gruppe von Pflegenden auf Intensivstationen zum Thema OMW befragt.

\section{Schlussfolgerung}

OMW stellen aus Sicht der Pflegenden in dieser Evaluationsstudie eine Möglichkeit zur unterstützenden Fortbildung im Praxisalltag von Pflegenden auf der Intensivstation dar. Es wird weitere Forschung benötigt, um einen Wissenstransfer empirisch zu belegen. Zusätzlich sollte zukünftig der Frage nachgegangen werden, inwieweit weitere an der Gesundheitsversorgung beteiligte Berufsgruppen von der Fortbildungsmethode profitieren können.

Danksagung Wir danken allen Pflegenden, die an dieser Studie teilgenommen haben. Besonderer Dank gilt auch den OMW-Autorinnen und -Autoren sowie den OMW-Beauftragten in allen Abteilungen. Tobias Becker danken wir für die Unterstützung bei der Erstellung des Fragebogens sowie der Datenverarbeitung.

Funding Open Access funding enabled and organized by Projekt DEAL.

Interessenkonflikt L. Krüger, T. Mannebach, F. Wefer und C. Bolte geben an, dass kein Interessenkonflikt besteht.

Open Access Dieser Artikel wird unter der Creative Commons Namensnennung 4.0 International Lizenz veröffentlicht, welche die Nutzung, Vervielfältigung, Bearbeitung, Verbreitung und Wiedergabe in jeglichem Medium und Format erlaubt, sofern Sie den/die ursprünglichen Autor(en) und die Quelle ordnungsgemäß nennen, einen Link 
zur Creative Commons Lizenz beifügen und angeben, ob Änderungen vorgenommen wurden.

Die in diesem Artikel enthaltenen Bilder und sonstiges Drittmaterial unterliegen ebenfalls der genannten Creative Commons Lizenz, sofern sich aus der Abbildungslegende nichts anderes ergibt. Sofern das betreffende Material nicht unter der genannten Creative Commons Lizenz steht und die betreffende Handlung nicht nach gesetzlichen Vorschriften erlaubt ist, ist für die oben aufgeführten Weiterverwendungen des Materials die Einwilligung des jeweiligen Rechteinhabers einzuholen.

Weitere Details zur Lizenz entnehmen Sie bitte der Lizenzinformation auf http://creativecommons.org/licenses/by/4.0/deed.de.

\section{Literatur}

Bray L (2016) Improving cranial ultrasound scanning strategy in neonates. BMJ Open Qual 5:u210346.w4219. https://doi.org/10. 1136/bmjquality.u210346.w4219

Brunnhuber M (2008) Weiterbildungsbereitschaft von Krankenpflegern. Empirische Untersuchung. Hamburg, Diplomica

Von Elm E, Altman DG, Egger M, Pocock SJ, Gøtzsche PC, Vandenbroucke JP (2008) The Strengthening the Reporting of Observational Studies in Epidemiology (STROBE) statement: guidelines for reporting observational studies. J Clin Epidemiol 61:344-349

Fessele K, Fandler M, Popp S (2018) Innovative Fortbildungskonzepte im Schichtdienst. Intensiv Notfallbehandl 43(3):83-88

Frick-Salzmann A (2017) Gedächtnis: Erinnern und Vergessen. Ein Blick ins Gehirn für Bildungs-, Gesundheits- und Sozialexperten. Springer, Wiesbaden

Haselinger-Baumann E, Lang G, Müller G (2015) Einfluss und Zusammenhang von Einstellung, Verfügbarkeit und institutioneller Unterstützung auf die Anwendung von Forschungsergebnissen in der pflegerischen Praxis-Ergebnisse einer explorativen quantitativen Querschnittstudie. Pflege 28(3):145-155

Jonker G, Agricola G, Bosman E, van Rijen H (2016) Digital interactive learning during idle time at work. Med Educ 50(11):1163-1164

Knoppik J (2004) Möglichkeiten von E-Learning in der Pflege. PrInterNet 6(1): 42-49

Krüger L (2017) Warten mit Sinn. intensiv 25(5):246-248

Krüger L, Mannebach T (2018) Wartezeiten zur Fortbildung nutzen. Pflegenintensiv 15(4):38-40

Lehnen T, Nydahl P, Krüger L (2019) Evaluation der Einminutenfortbildung. Schnelle und effektive Wissensvermittlung. Pflegenintensiv 16(2):48-50

Löwenstein M (2016) Förderung der Lernkompetenz in der Pflegeausbildung. Lehr-Lern-Kultur durch Lernportfolios verändern. Springer, Wiesbaden

Mayer H (2015) Pflegeforschung anwenden. Elemente und Basiswissen für das Studium, 4. Aufl. facultas, Wien

Rowlinson J (2014) The one minute wonder network. Clin Teach 11:332-335

Schmidt B, Krüger L (2016) Lernen in nur einer Minute. intensiv 24(5):258-259

Schniering S, Baumeister A, Darmann-Finck I (2015) „Eher kürzere Handlungsanweisungen?" Anforderungen an die Konzeption einer problemorientierten Wissensdatenbank für Pflegende. Pflegewissenschaft 17(6):335-344

Weigl R (2016) Quantitative Messverfahren. In: Ritschl V, Weigl R, Stamm T (Hrsg) Wissenschaftliches Arbeiten und Schreiben. Verstehen, Anwenden, Nutzen für die Praxis. Springer, Berlin, S $160-192$

Hinweis des Verlags Der Verlag bleibt in Hinblick auf geografische Zuordnungen und Gebietsbezeichnungen in veröffentlichten Karten und Institutsadressen neutral. 